\title{
Can regional training courses reduce interobserver variability?
}

\author{
Erick Alexanderson-Rosas, $M D,{ }^{a, b}$ and Alondra Flores-García, $M^{a}$ \\ a Nuclear Cardiology Department, Instituto Nacional de Cardiologia "Ignacio Chavez", Mexico \\ City, Mexico \\ b Departamento de Fisiologia, Facultad de Medicina, Universidad Nacional Autonoma de \\ Mexico, Mexico City, Mexico
}

Received Sep 15, 2018; accepted Sep 17, 2018

doi:10.1007/s12350-018-1453-y

Cardiovascular disease (CVD) is the principal cause of death worldwide. Noninvasive studies have been used for the evaluation of CVD. Ensuring an accurate diagnosis of CVD requires well-trained and qualified professionals. IAEA has implemented regional training courses which are mainly aimed at professionals from countries with less economic development in order to raise their professional level so that it is in accordance with international standards and thus be able to homogenize the practice of nuclear cardiology globally.

Key Words: Image interpretation • SPECT • Outcomes research

\section{See related article, pp. 465-478}

Cardiovascular disease (CVD) is the principal cause of death worldwide, and its high rates of incidence, prevalence, and associated comorbidities impose severe economic impacts on health systems. For this reason, it has become important to establish increasingly effective strategies of prevention, diagnosis, and treatment.

In recent decades, noninvasive studies have been used more and more frequently for the evaluation of CVDs, since the advancement of technology allows them to be produce more precise results every time, so that an early and accurate diagnosis can be made.

Myocardial perfusion imaging (MIP) with Single Photon Emission Computed Tomography (SPECT) represents $12-13 \%$ of all nuclear medicine procedures. Its high negative predictive value allows such a high degree of accuracy that it can rule out the presence of

Reprint requests: Erick Alexanderson-Rosas, MD, Nuclear Cardiology Department, Instituto Nacional de Cardiologia "Ignacio Chavez", Mexico City, Mexico; alexandersonerick@gmail.com J Nucl Cardiol 2020;27:479-80.

$1071-3581 / \$ 34.00$

Copyright (C) 2018 American Society of Nuclear Cardiology.
CVD when we have a prediction of a normal study. Therefore, it can be used to effectively diagnose, stratify the patient, and provide the qualitative prognosis of cardiac events in the future.

However, the economic situation of each country is fundamental to ensure that its population has access to the appropriate technological tools, but the distribution of this technology worldwide is not homogeneous and does not always respond to the specific health needs of the country. The developing countries present significant difficulties, as the installation of nuclear medicine centers requires an infrastructure of expensive equipment and materials as well as the continuous investment for the acquisition of radiotracers, drugs, licenses of processing software, and equipment maintenance. In addition, other difficulties are the lack of qualified personnel and the difficulties that their training and continuous updating represent. This last item is just one of the most important aspects since the competent interpretation of the MPI has a great impact on the clinical approach to the patient.

The MPI, like any other diagnostic noninvasive method, can present intra- and interobserver variabilities, as well as their experience. In view of MPI being a semiquantitative method of analysis, this possibility is reduced, but it is not eliminated, and for this reason, the International Atomic Energy Agency (IAEA) has established regional training courses (RTCs) which are 
mainly aimed at professionals from countries with less economic development. The objective of the RTCs is to provide educational tools to these groups, in order to raise their professional level so that it is in accordance with international standards and thus help to homogenize the practice of nuclear cardiology across the world and ensure qualitative interpretation of the MPI.

It is important to point out that each strategy must be accurately evaluated. The IAEA, through Integrated Monitoring and Assessment Programme (iMAP), has been given the task to evaluate the efficiencies of RTCs in the practice of nuclear cardiology by measuring their effectiveness through interobserver variability at the moment of providing a diagnosis. Dondi et al. ${ }^{1}$ in their study on inter-reader variability of SPECT MPI readings in low- and middle-income countries provide the results of an audit, and they concluded that the role of training implemented by the IAEA is fundamental to educational activities having a positive impact on the diagnostic interpretation of MPI. ${ }^{2}$
The IAEA through the establishment of RTCs has facilitated the equitable sharing of knowledge in nuclear cardiology, with a view to ensure that professionals from developing countries have access to quality education for the optimal performance of their functions.

\section{Disclosure}

The authors have no conflict of interest to disclose.

\section{References}

1. Dondi M, Kashyap R, Paez D, Pascual T, Zaknun J, Mut Bastos F, Pynda Y. Trends in nuclear medicine in developing countries. J Nucl Med. 2011;56:16S-23S.

2. Maurizio Dondi, Thomas Pascual, Diana Paez. Improving Nuclear Medicine practices in cardiology in the eeemerging economies: Role of the International Atomic Energy Agency. Int Journ Cardiovasc Scim. 2018;31:71-8. 